\title{
Extraction of Signal Waveform Feature Based on Bispectrum
}

\author{
Digang Wang1*, Xiaoqun Zhao ${ }^{1}$, Yang Zhang1 \\ 1 Tongji University, No. 4800, CaoAn Road, Jiading District, Shanghai, China. \\ * Corresponding author. Tel.: +86 18502134325; email: appana@163.com \\ Manuscript submitted May 23, 2014; accepted December 8, 2014. \\ doi: 10.17706/ijcce.2015.v4.386
}

\begin{abstract}
This paper presents a new method of feature extraction for signal waveform. Selection of a feature extraction method is probably the single most important factor in achieving high recognition performance. Over the past four decades, considerable work has been done in the area of power spectrum estimation. However, a problem with this method is that it is phase blind. Situations arise in science and engineering whereby signal analysts are required to look beyond second-order statistics and analyze a signal's Higher-Order Statistics (HOS). In this paper, bispectrum is used to extract the feature of signal. Feature of the signal can be extracted by selecting the eigenvector whose corresponding eigenvalue's module is the largest as the template of recognition. The experiment being made by our research group suggests that recognition accuracy rate of bispectrum-method can be no less than 90 percent in additive white Gaussian noise channel when SNR (Signal to noise rate) is no less than 8dB .
\end{abstract}

Key words: Eigenvalue and eigenvector, feature extraction, higher order spectrum (HOS), signal processing, signal recognition.

\section{Introduction}

For the last forty years the use of bispectrum has become a growing field of interest. Classification of signal modulation type has received increasing attention as it can be used in identifying monitoring, detecting transmissions, spectrum management and electronic warfare.

Automatic communication signals recognition plays an important role for various applications. Automatic signal classification techniques, usually, are divided in two principle techniques: decision theoretic (DT) approaches and pattern recognition (PR) approaches [1]. The major drawbacks of DT methods are their too high computational complexity, lack of robustness to the model mismatch as well as careful analysis that are required to set the correct threshold values. Pattern recognition approaches, however, do not need such careful treatment. They are easy to implement. PR methods can be further divided in two main subsystems: the feature extraction subsystem and the classifier subsystem [2].

Traditionally, a zero-mean random process is described via its second-order statistics, i.e. its autocorrelation function or equivalently its power spectrum. In general, these measures are only a partial description of an arbitrary random process. Decision theoretical approaches require a statistical description of the signals and usually involve the definition of likelihood ratio tests [3]. As a result, they are usually extremely complex to compute. High-order cumulants is one of the most effective methods in statistical pattern recognition approaches, because it can remove the influence of Gauss noise completely in theory. The higher-order statistics (HOS) [4] of primary concern is the third-order spectrum, the 
'bispectrum'. The bispectrum [5] of a signal is the Fourier transform of its triple correlation. This is the simplest of HOS [6]. While second-order statistics [7] (e.g., correlation function, power spectrum, etc.) do not provide any information about the phase of the signal, third-order statistics [8] (e.g., triple correlation, bispectrum, etc.) allow the recovery of the phase of the signal. The bispectrum provides information in cases when the random process has a 'skewed' distribution [9] (skewness is a measure of asymmetry of data around the mean).

In this work, we combine bispectrum with eigenvalue decomposition method. As the eigenvalues of practical signal's bispectrum have an interesting property that most of absolute value of the eigenvalues are much smaller than the rest eigenvalues [10]. According to the property of eigenvalue, it is reasonable to believe that the eigenvector of the eigenvalue whose absolute value is the largest can reflect the feature of the signal [11]. As a result, we choose the eigenvector of the eigenvalue whose absolute value is the largest as the recognition template to distinguish the signal from other types of signal [12].

This paper is organized in the following manner. In the Section 2, the approach of modulation signal recognition will be presented. In Section 3, we will introduce the definition of bispectrum. The prosperity of eigenvalue of bispectrum is presented in Section 4. Finally simulations are provided in Section 5. Conclusions are drawn in Section 5.

\section{Definition of Bispectrum}

\subsection{Base Definition}

Although there are several different methods for estimating second-order spectrum (Bispectrum), the direct method is selected to estimate bispectrum in this paper. The bispectrum is defined as the triple product:

$$
B\left(f_{1}, f_{2}\right)=X\left(f_{1}\right) X\left(f_{2}\right) X^{*}\left(f_{1}+f_{2}\right)
$$

where $B$ is the bispectrum evaluated at frequencies $f_{1}$ and $f_{2}$, X denotes the Fourier transform of the signal, and ${ }^{*}$ denotes the complex conjugate.

The Fourier transform is a complex quantity, and so is the bispectrum. Bispectra fall in the category of higher-order spectra, or polyspectra and provide supplementary information to the power spectrum. The third order polyspectrum (bispectrum) is the easiest to compute, and hence the most popular. From complex multiplication, the magnitude of the bispectrum is equal to the product of the magnitudes of each of the frequency components, and the phase of the bispectrum is the sum of the phases of each of the frequency components.

A similar analysis applied to the fourth order leads to a definition of the trispectrum (for a non-stochastic signal) as

$$
B_{x x x}\left(f_{1}, f_{2}, f_{3}\right)=X\left(f_{1}\right) X\left(f_{2}\right) X\left(f_{3}\right) X^{*}\left(f_{1}+f_{2}+f_{3}\right)
$$

\subsection{Characteristics of Higher-Order Spectrum (HOS)}

Gaussian processes are intimately connected with the study of second-order methods since it is only Gaussian processes which can be completely described by their second-order properties. Hence, if a process is Gaussian then the HOS contain no new information, i.e. no information that cannot be obtained from the second-order statistics. This leads us to one desirable property of HOS, namely that one would like them to be identically zero for a Gaussian process.

The first of the HOS is the third-order spectrum, given the name bispectrum. This quantity has received most attention in the literature since it is the simplest of the HOS. However, the bispectrum only yields 
information in cases where the random process has a skewed distribution. In a significant number of physical problems, systems are symmetrical and yield unskewed output signals. In these circumstances, the bispectrum is an uninformative measure. One of the aims of this paper is to show how the concepts associated with the bispectrum carry over to the fourth-order spectrum, referred to as the trispectrum, and to discuss how the trispectrum can be used to analyze symmetric non-linearities.

\subsection{The Relationship between Cumulant Functions and HOS}

The Wiener-Khintchine theorem states that the autocorrelation function and power spectrum are related via the Fourier transform, i.e.

$$
B_{x x}(f)=\int_{-\infty}^{\infty} R_{x x}(\tau) e^{-j 2 \pi f \tau} d \tau=\int_{-\infty}^{\infty} C_{x x}(\tau) e^{-j 2 \pi f \tau} d \tau
$$

Similar results relate HOS to cumulant and moment functions. The bispectrum can be defined as the double Fourier transform of the cumulant (or moment) function:

$$
B_{x x x}\left(f_{1}, f_{2}\right)=\int_{-\infty}^{\infty} \int_{-\infty}^{\infty} C_{x x x}\left(\tau_{1}, \tau_{2}\right) e^{-j 2 \pi\left(f_{1} \tau_{1}+f_{2} \tau_{2}\right)} d \tau_{1} d \tau_{2}
$$

In the trispectral case, one has to take more care, since the moment and cumulant functions are different, which leads to two possible definitions of the trispectrum:

$$
B_{x x x x}\left(f_{1}, f_{2}, f_{3}\right)=\int_{-\infty}^{\infty} \int_{-\infty}^{\infty} \int_{-\infty}^{\infty} C_{x x x x}\left(\tau_{1}, \tau_{2}, \tau_{3}\right) \mathrm{e}^{-j 2 \pi\left(f_{1} \tau_{1}+f_{2} \tau_{2}+f_{3} \tau_{3}\right)} d \tau_{1} d \tau_{2} d \tau_{3}
$$

\subsection{Matric Form of Bispectrum}

From the definition of bispectrum, we can find that bispectrum is two-dimensional signal. So, a matrix can be used to describe the bispectrum (6):

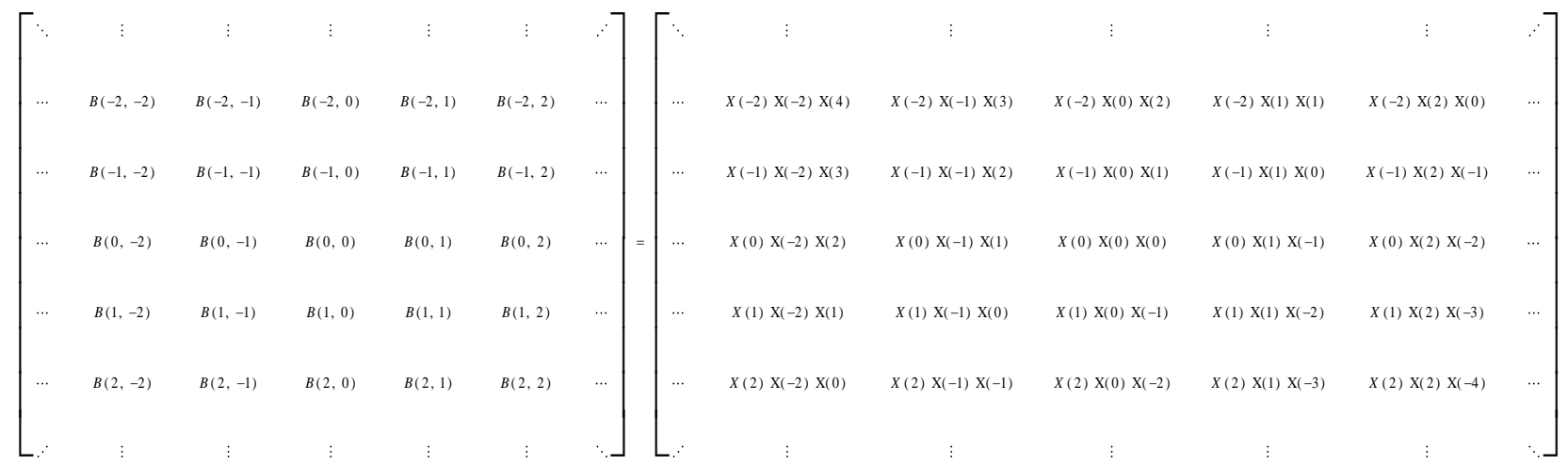

Such definition of bispectrum is based on an assumption that the signal is infinite. However, the actual signal is often truncated and the bispectrum of signal is also finite. In this paper, the size of bispectral matrix is defined as $128 \times 128$ matrix. As a result, bispectrum can also be expressed as (7): 


\subsection{Symmetry Properties of Bispectra and Trispectra}

Just as the continuous power spectrum has symmetrical properties, so do the continuous bispectrum and trispectrum. It is only necessary to evaluate the bispectrum and trispectrum in the principal domain or non-redundant area, as all other regions can be found by symmetrical transformations of this area. In addition to the symmetrical properties of the continuous bispectrum and trispectrum, the discrete versions, like the discrete power spectrum, are periodic.

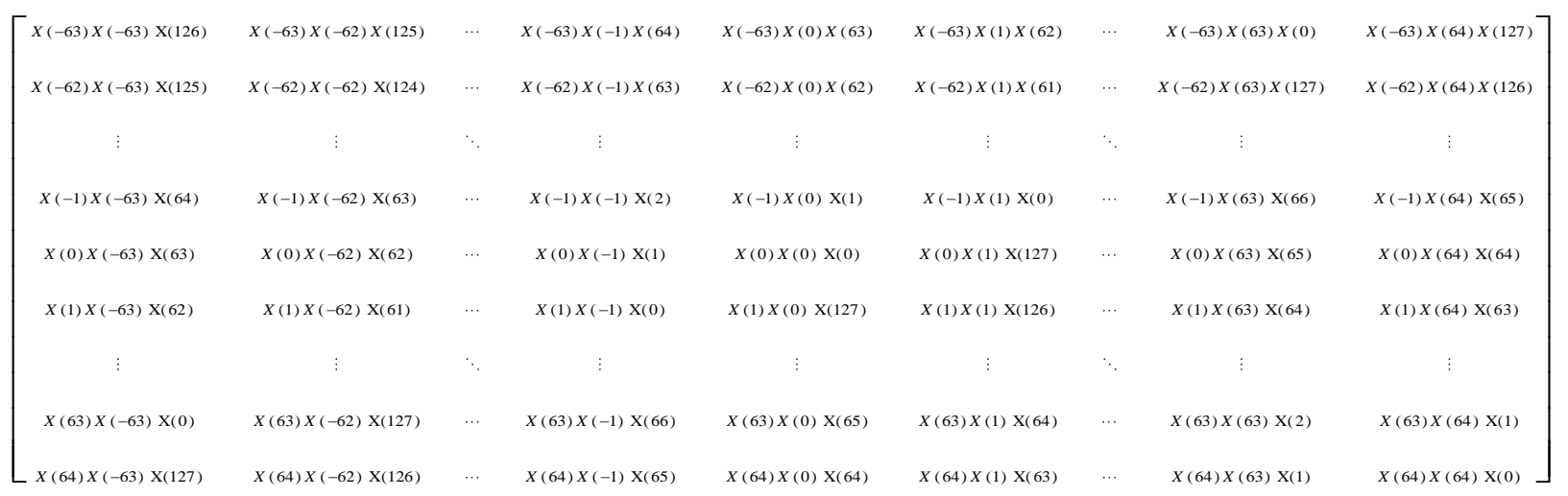

Knowledge of the bispectrum in the triangular region $f 1, f 2>0$, and $f 2>f 1$ is enough for a complete description of the continuous bispectrum. There are in fact 12 regions of symmetry in the continuous bispectrum. As the discrete Fourier transform is periodic, the discrete bispectrum is also periodic which means that there are added symmetries for the discrete bispectrum, and it can be shown that it is only necessary to estimate the discrete bispectrum in the region $0<f 2<f 1$, and $f 1+f 2<f s / 2$. This region is known as the principal domain and can be further subdivided into two regions. There are shown by the two triangles in Fig. 1 (bispectrum), the inner triangle and the outer triangle.
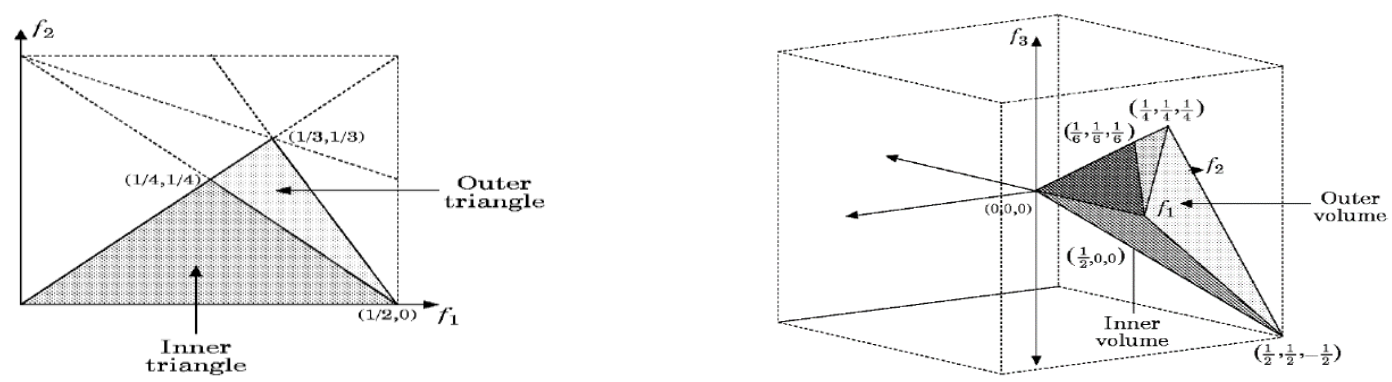

Fig. 1. Principal domain of the bispectrum and the trispectrum.

The discrete trispectrum has 96 regions of symmetry. The principal domain is shown in Fig. 1 (trispectrum). Like the bispectrum, it is divided into two regions: an inner volume (shown in dark grey); and outer volume (shown in light grey). Referring to Fig. 1 (trispectrum), both the inner and outer volume can be subdivided into two further regions: above and below the $f 3=0$ plane. Above the plane, all the frequency indices are positive and so the sum of three frequencies is equal and opposite of the fourth. Below the plane, only two of the first three frequencies are positive and so this region contains interactions where the sum of two frequencies is equal and opposite to the sum of the other two.

\section{Feature Extraction}




\subsection{Property of Eigenvalue of Modulation Signal's Bispectrum}

In order to extract the feature of signal, eigenvalue and eigenvector will be calculated. The eigenvalue equation can be defined as follow

$$
|\lambda E-B|=0
$$

where $E$ is a unit matrix whose size is the same as the matrix $B$ and $B$ is a bispectral matrix whose size is $128 \times 128$. After doing a large numbers of experiments, we unexpectedly find that the eigenvalue of the matrix B has an interesting property that most of the eigenvalues are much smaller than the rest of the eigenvalues.

Through large number of experiment, we find that there are only a few eigenvalues which are comparatively very large while the rest eigenvalues are close to zero (Fig. 2). Of course, such properties will not be true when the signal spectrum accounts for the most part of frequency domain.

\subsection{Scope of Application of Our Recognition Algorithm}

Fig. 3 is the spectrum of 2ASK, 2FSK, 2PSK, 16QAM, 4FSK, GMSK which are drawn to state the above theory clearly.

From the Fig. 3, we can find that the signal spectrum just accounts for small part of total frequency domain. In the actual engineering, the sample-rate will always be twice larger than maximum of signal frequency. Moreover, practical signals in engineering are often band-pass signals, which will result in that signal spectrum just accounts for small part of total frequency domain. As a result, our experiment corresponds to the reality.
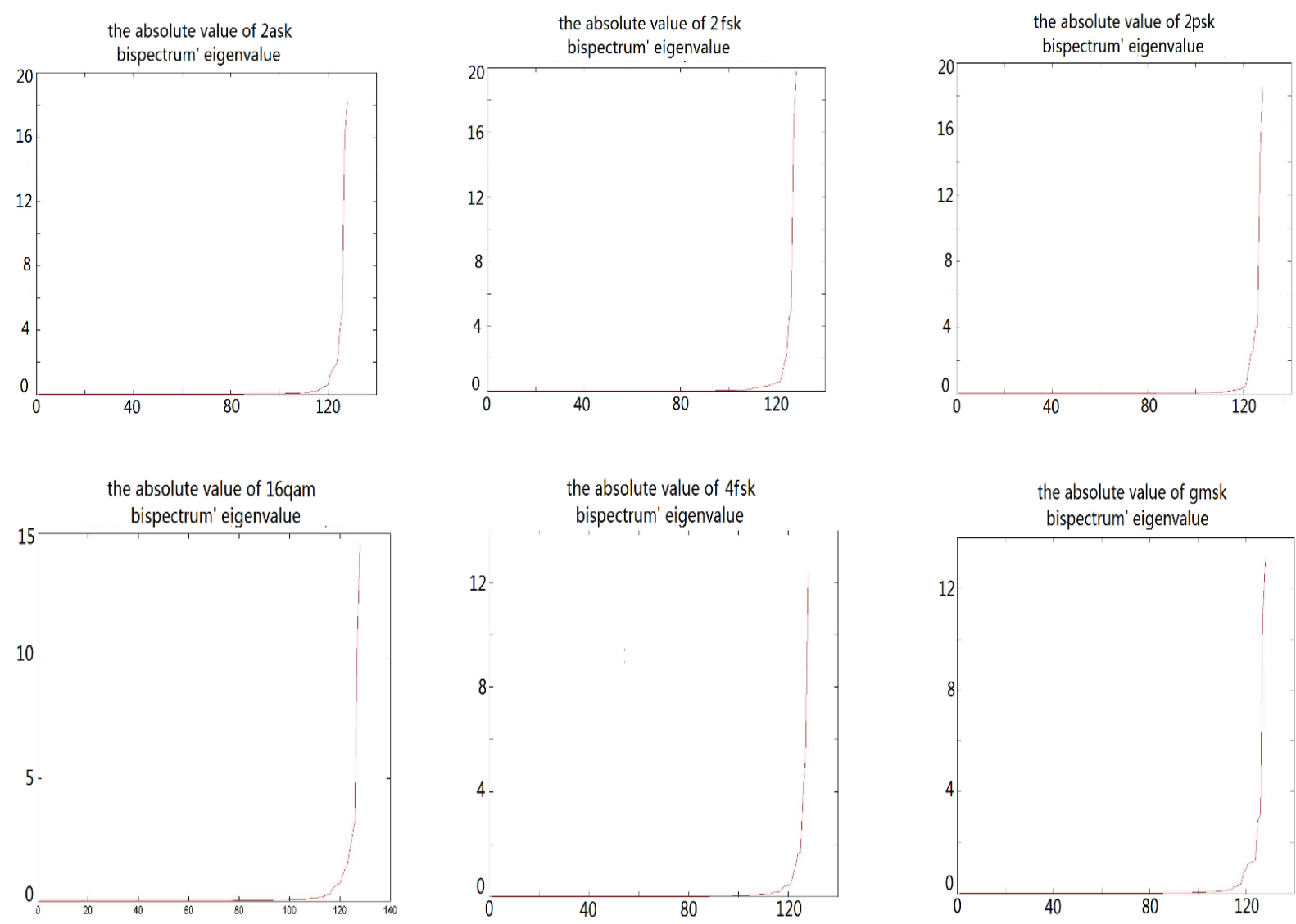

Fig. 2. The absolute value of eigenvalue of bispectrum of 2ASK, 2FSK, 2PSK, 16QAM, 4FSK, GMSK. (The absolute value of eigenvalue is arranged from smallest to largest). 

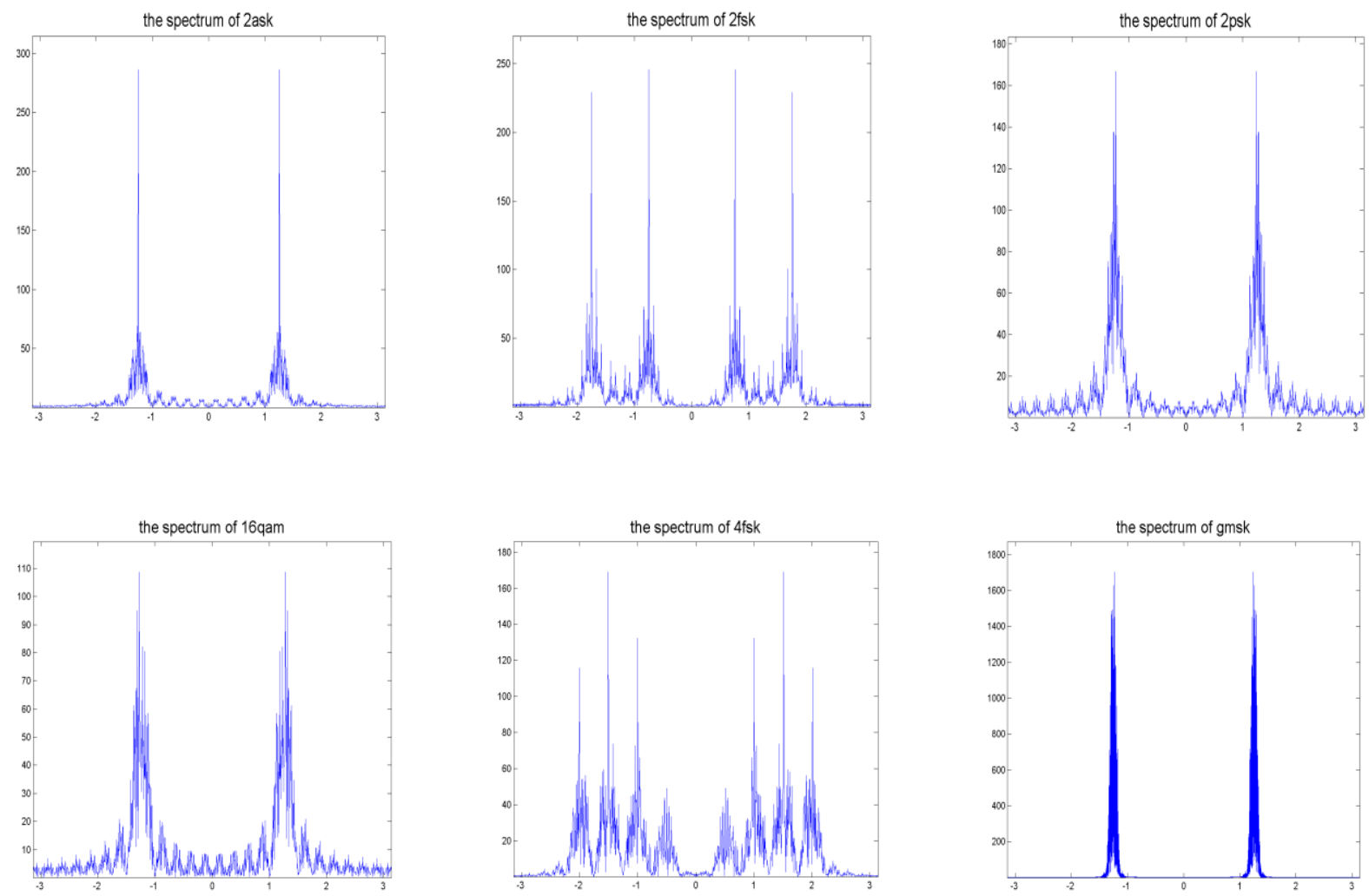

Fig. 3. The spectrum of 2ASK, 2FSK, 2PSK, 16QAM, 4FSK, GMSK.

\subsection{The Method of Modulation Signal Recognition Based on Bispectrum and Eigenvector Extraction}

As the eigenvalue of bispectrum of modulation signals always have such property as Fig. 2 shows that, the eigenvector of eigenvalue whose absolute value is largest can be regarded as the template which reflects the essence of signal to distinguish the signal from the other types of signal. In the experiment, six kinds of modulation signal (2ASK, 2FSK, 2PSK, 16QAM, 4FSK and GMSK) are chosen to simulate the process of signal recognition. Then, eigenvalue and eigenvector of bispectral matrix are calculated and the eigenvector whose eigenvalue's absolute value is the largest is chosen as the template of signal.

The eigenvalue equation for a matrix $A$ is:

$$
B v-\lambda v=\overrightarrow{0} \quad \text { or } \quad(B-\lambda E)=\overrightarrow{0}
$$

where $\mathrm{E}$ is the identity matrix whose size is the same as B and B is a bispectral matrix whose size is $128 \times 128$. Then, we will get the formal definition of characteristic polynomial of matrix B:

$$
\mathrm{p}_{\mathrm{B}}(\lambda)=\operatorname{det}(\lambda E-B)
$$

and the characteristic equation:

$$
\operatorname{det}(\lambda E-B)=0
$$

In the complex domain, the characteristic polynomial of an $n \times n$ matrix B, being a polynomial of degree $n$, has exactly n complex roots. More precisely, it can be factored into the product of $\mathrm{n}$ linear terms: 


$$
\operatorname{det}(B-\lambda E)=\left(\lambda_{1}-\lambda\right)\left(\lambda_{2}-\lambda\right) \operatorname{L}\left(\lambda_{n}-\lambda\right)
$$

where each $\lambda \mathrm{i}$ is a complex number. The numbers $\lambda 1, \lambda 2 \ldots \lambda \mathrm{n}$ are roots of the polynomial and are precisely the eigenvalues of $\mathrm{B}$. To find eigenvectors $\mathrm{v}=[\mathrm{v} 1, \mathrm{v} 2 \ldots \mathrm{vn}]$ corresponding to an eigenvalue $\lambda$, we simply solve the system of linear equations given by:

$$
(B-\lambda E) \overrightarrow{\mathrm{v}}=\overrightarrow{0}
$$

Using the above method, we will calculate the eigenvector $\mathrm{v}$ corresponding to an eigenvalue $\lambda$ whose absolute value is largest as the template which reflects the essence of signal to distinguish the signal from the other types of signal.

\section{Simulation and Results}

Considering the above character of bispectral eigenvalue, the eigenvector of eigenvalue whose absolute value is largest can be regarded as the template which reflects the essence of signal to distinguish the signal from the other types of signal. As a result, the following method is designed to realize modulation signal recognition.

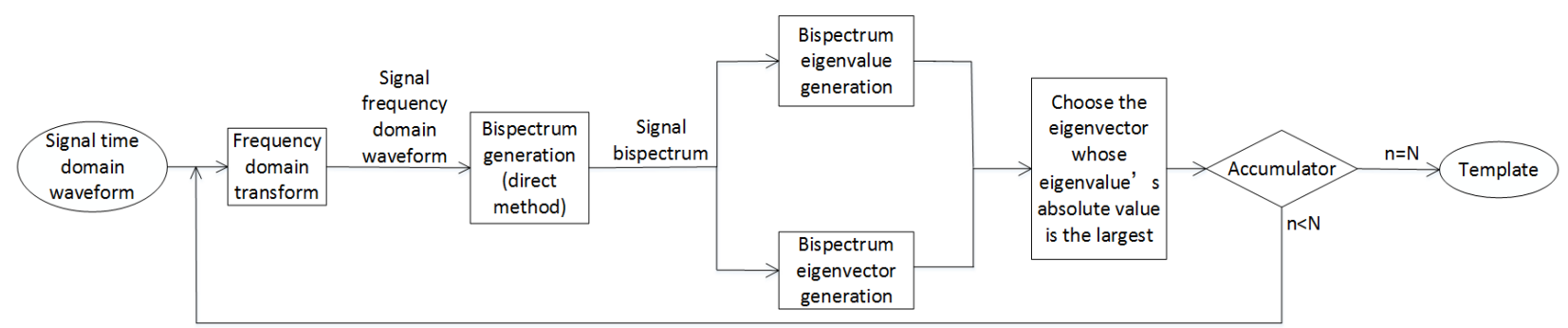

Fig. 4. The flow chart of template training.

The flow chart of template training (Fig. 4) is made to describe the process of template training. In the first place, computer is used to simulate a kind of modulation signal. In the experiment, six kinds of modulation signal are chosen to simulate the process of signal recognition. In the second place, signal bispectrum is calculated through the direct method. In the experiment, signal bispectrum is expressed as a $128 \times 128$ matrix. In the third place, eigenvalue and eigenvector of bispectral matrix are calculated and the eigenvector whose eigenvalue's absolute value is the largest is chosen as the template of signal. The above process will rum for five hundred times so as to get the average of template which serves as the final template to recognize the type of signal.

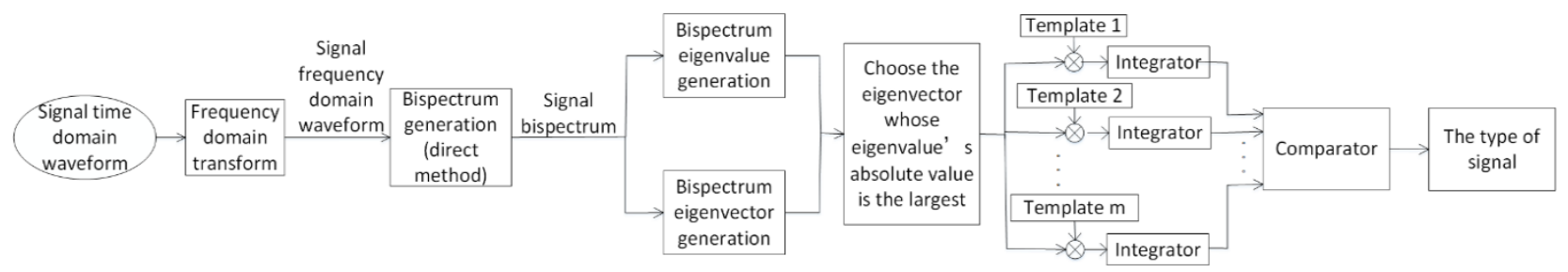

Fig. 5. The flow chart of signal recognition.

The flow chart of signal recognition (Fig. 5) is made to describe the process of signal recognition. In the experiment, six modulation signal (2ASK, 2FSK, 2PSK, 16QAM, 4FSK and GMSK) are chosen as the objects to be recognized. Firstly, the eigenvalues and eigenvectors the signal to be recognized are calculated by the method like template training. Secondly, the eigenvector of eigenvalue whose absolute value is the largest among all the eigenvalue will be extracted and be multiplied with the six templates which have been trained 
before. The product of two signals will be integrated so as to get the correlation coefficient between practical signal and template signal. Six correlation coefficients will be calculated by the method of mutual correlation in the same way and be sent to the comparator in order to find out the largest correlation coefficient. Finally, the template with the largest correlation coefficient will be regarded as the correct type of signal.

In this experiment, we choose six signals (2ASK, 2FSK, 2PSK, 16QAM, 4FSK and GMSK) to be recognized in different SNR $(5 \mathrm{~dB}, 8 \mathrm{~dB}, 12 \mathrm{~dB}, 16 \mathrm{~dB}, 20 \mathrm{~dB})$. At training process, ten thousand groups of signal of each modulation type are used to calculate the template of signal. At test process, one thousand groups of signal of each modulation type are used to calculate the accuracy of recognition.

In the Table I, accuracy rate is no less than 90 percent when SNR is no less than 8dB. The recognition accuracy rate of signal of 2ASK, 2PSK and 16QAM decreased rapid when the signal channel is deteriorating. The possible reason is that the correlation coefficient among 2ASK, 2PSK and 16QAM are relatively larger than others.

Table I. Accuracy of the Recognition Method (\%)

\begin{tabular}{ccccccc}
\hline \hline SNR(dB) & 2 ASK & 2 FSK & 2PSK & 16QAM & 4 FSK & GMSK \\
\hline 5 & 92.0 & 96.2 & 85.2 & 89.1 & 98.2 & 90.0 \\
8 & 97.2 & 98.9 & 90.1 & 93.3 & 99.2 & 94.3 \\
12 & 98.6 & 99.9 & 94.3 & 97.0 & 99.0 & 94.7 \\
16 & 99.2 & 100 & 96.2 & 98.5 & 100 & 99.6 \\
20 & 100.0 & 100.0 & 100.0 & 100.0 & 100.0 & 100.0 \\
\hline \hline
\end{tabular}

\section{Conclusion}

We present a new recognition algorithm which combines the bispectrum with eigenvalue extraction. The proposed algorithm is capable of recognizing different modulation schemes with high accuracy at low SNRs. In order to reduce the dimension of the signal bispectrum, the largest eigenvector whose corresponding eigenvalue's module is the largest is selected as the template of recognition. Our experimental results confirm that modulation signal recognition using bispectrum and eigenvector as the template of recognition is indeed possible and viable. Our classifier has high modulation recognition performance when the SNR is not lower than $8 \mathrm{~dB}$.

However, there is a foible in this method that it is relatively complicated in the training process, but this method not only reduces the dimension of bispectrum and extract the feature of signal but also overcomes the shortage of the phase blind of power spectrum. Another advantage of our method is that any Gaussian signal and narrow-band Gaussian signal will be filtered by bispectrum, which increases the recognition rate in low SNRs. Simulations justify the efficiency of this classification algorithm. In future work, we plan to extend our techniques to incorporate other types of classification methods to increase the recognition rate in other types of noise.

\section{References}

[1] Fu, K. S., \& Rosenfeld, A. (1976). Pattern recognition and image processing. IEEE Transactions on Computers, 100(12), 1336-1346.

[2] Bishop, C. M., \& Nasrabadi, N. M. (2006). Pattern Recognition and Machine Learning. New York: Springer.

[3] Jain, A. K., Duin, R. P. W., \& Mao, J. (2000). Statistical pattern recognition: A review. IEEE Transactions on 
Pattern Analysis and Machine Intelligence, 22(1), 4-37.

[4] Dobre, O. A., Abdi, A., \& Bar-Ness, Y, et al. (2007). Survey of automatic modulation classification techniques: classical approaches and new trends. Communications, 1(2), 137-156.

[5] Orlic, V. D., \& Dukic, M. L. (2009). Automatic modulation classification algorithm using higher-order cumulants under real-world channel conditions. Communications Letters, 13(12), 917-919.

[6] Boutte, D., \& Santhanam, B. (2009). A hybrid ICA-SVM approach to continuous phase modulation recognition. Signal Processing Letters, 16(5), 402-405.

[7] Hasselmann, K., Munk, W. H., \& MacDonald, G. J. F. (1963) Bispectra of ocean waves. Time Series Analysis, 125-139.

[8] Pawar, S. U., \& Doherty, J. F. (2011). Modulation recognition in continuous phase modulation using approximate entropy. IEEE Transactions on Information Forensics and Security, 6(3), 843-852.

[9] Kavalov, D., \& Kalinin, V. (2002). Neural network surface acoustic wave RF signal processor for digital modulation recognition. IEEE Transactions on Ultrasonics, Ferroelectrics and Frequency Control, 49(9), 1280-1290.

[10] Xu, J. L., Su, W., \& Zhou, M. C. (2010). Software-defined radio equipped with rapid modulation recognition. IEEE Transactions on Vehicular Technology, 59(4), 1659-1667.

[11] Coulon, M., Tourneret. J-Y., \& Swami, A. (1999). Detection and classification of spectrally equivalent processes using higher order statistics. IEEE Transactions on Signal Processing, 47(12), 3326-3335.

[12] Chandran, V., \& Elgar, S. (1990). Bispectral analysis of two-dimensional random processes. Acoustics, IEEE Transactions on Speech and Signal Processing, 38(12), 2181-2186.

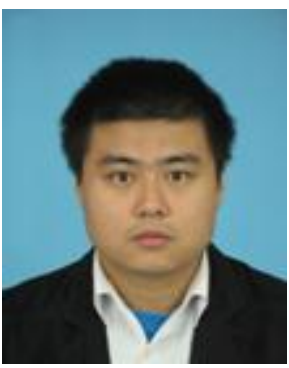

Digang Wang was born in Shanghai, China, on 30 March, 1988. He received a B.S. degree in communication engineering in Tongji University, Shanghai, China, in 2010. He is currently pursuing the M.S. degree with the Department of Information and Communication Engineering, Tongji University, Shanghai, China.

His current research interests include higher order spectrum analysis, communication and signal processing and coding theory.

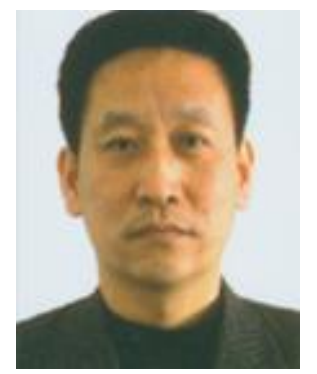

Xiaoqun Zhao was born in Heilongiang Province, in March, 1962. He received the Ph.D. degree in communication and information system from Harbin Institute of Technology. He is currently a distinguish professor and doctoral supervisor of the Department of Information and Communication Engineering, Tongji University, Shanghai, China.

He has authored or co-authored more than 100 papers in refereed journals and conference proceedings, and has authored and co-authored three books. His current research interests include modern spectrum estimate, speech encoding, underwater acoustic communication.

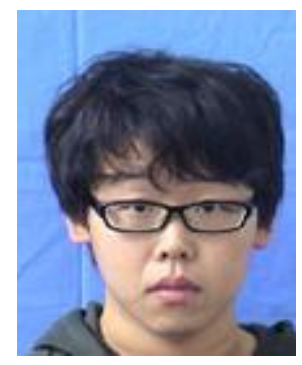

Yang Zhang received a B.S. degree in communication engineering in Tongji University, Shanghai, China, in 2012. He is currently pursuing the Ph.D. degree with the Department of Control Science and Engineering, Tongji University, Shanghai, China.

His current research interests include higher order spectrum analysis, communication and signal processing and coding theory. 